\title{
Superficial siderosis of the central nervous system
}

\author{
Mayuri Govind, MB ChB, FCRad Diag (SA) \\ Department of Radiology, Inkosi Albert Luthuli Central Hospital, Durban

\section{J Maharajh, MB ChB, FC Rad Diag (SA), MMed \\ Department of Radiology, Nelson R Mandela School of Medicine, and University of KwaZulu-Natal, Durban}

\section{Abstract}

The term superficial siderosis is used to describe the haemosiderin deposition on the surface of the brain, spinal cord, brainstem and cranial nerve leptomeninges following recurrent subarachnoid haemorrhage. The concern is the cytotoxic nature of the haemosiderin on the underlying tissue causing slow but progressive and irreversible neurological dysfunction. Removal of the source of bleeding has little effect on the progression of clinical deterioration but halting the chronic subarachoid haemorrhage will reduce the iron load in the CSF and hence the cytotoxic risk. We present a case of superficial siderosis presenting with hearing loss and cerebellar signs highlighting the imaging findings.
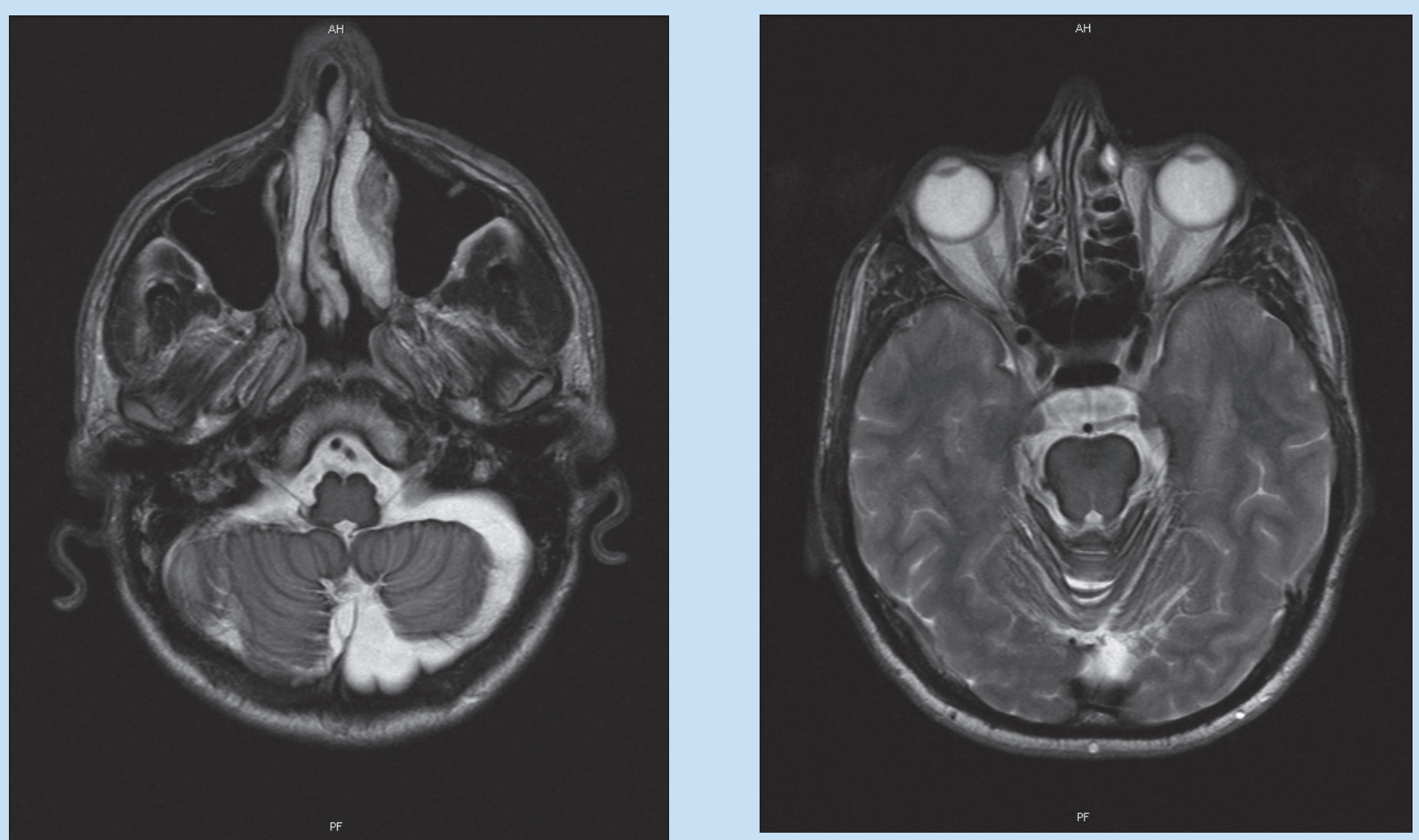

Figs 1 and 2. MRI - T2W axial images demonstrating hypointense lining along the pial surface of the cerebellum indicative of haemosiderin deposition and brainstem.

\section{Introduction}

Superficial siderosis describes the hemosiderin deposition on the surface of the brain, brainstem, cranial nerves and spinal cord following recurrent subarachnoid haemorrhage. The diagnosis can be made on MRI. The gradient echo sequence (GRE) best demonstrates the haemosiderin deposition on the surface of the brain ${ }^{1}$ and should prompt one to search for the cause of haemorrhage in order to prevent the irreversible neurotoxic effects on the underlying cortex.

\section{Case report}

This 44-year-old patient presented with a history of night sweats and malaise. He was observed to be inco-ordinate, had weakness of the left leg and changes in speech. Of significance is a history of two episodes of sudden loss of consciousness 6 months previously. He complained of hearing loss in his left ear.

On examination, he had upper limb cerebellar signs of intention tremor and disdiadocokinesis. He was also ataxic, demonstrated heel to shin ataxia, broad-based gait and was unable to tandem walk. The Rhomberg test was negative. The hearing loss was determined to be of the sensorineural type. 


\section{CASE REPORT}
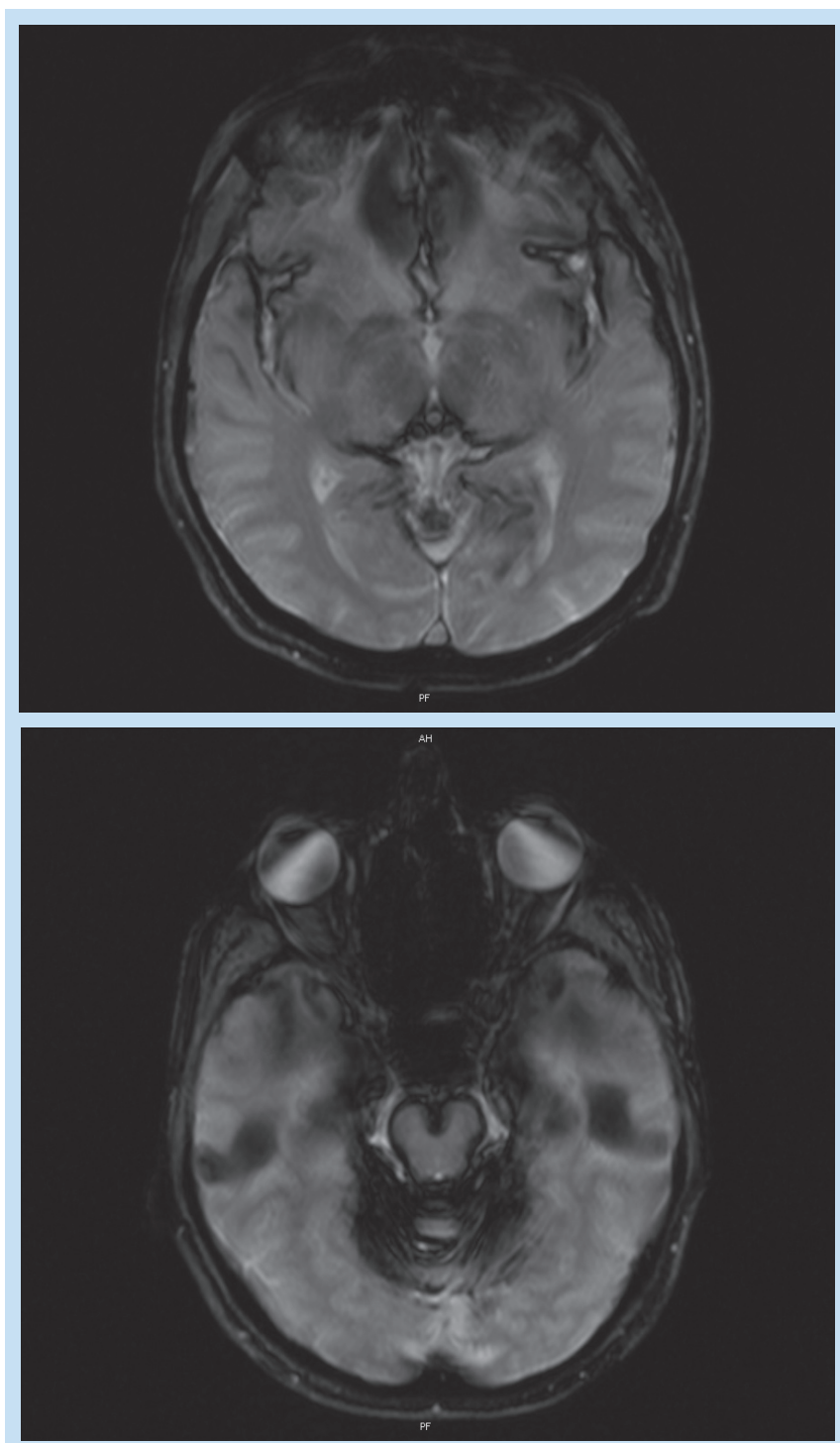

Figs 3 and 4. MRI - GRE axial sequence confirms the blood degradation products seen as hypointensity along the pial surface of the sylvian fissures bilaterally. 'Blooming' of the hypo-intense lining of the brainstem is present when compared to the T2 images.

Laboratory investigations were normal including full blood count, iron studies, clotting profile, thyroid function test and erythrocyte sedimentation rate. His cerebrospinal fluid (CSF) was xanthochromic and contained abundant red blood cells.

The MRI scans are diagnostic of superficial siderosis of the brain and spinal cord (Figs 1-5). There is 'staining' or lining of the meningeal surface of predominantly the cerebellum, brainstem and cord, but this may also include the sylvian fissures more superiorly. This is seen on T1, T2 and gradient echo sequences. Magnetic resonant angiography (MRA) and magnetic resonant venography (MRV) were normal. No intraparenchymal haemorrhage was noted.

\section{Discussion}

The term superficial siderosis is used to describe the haemosiderin deposition on the surface of the brain, spinal cord, brainstem and cranial nerve leptomeninges ${ }^{1}$ following recurrent subarachnoid haemorrhage.

Patients present with a triad of progressive bilateral sensorineural hearing loss (95\%), cytotoxic consequences on the cerebellum of limb and gait ataxia and myelopathy. ${ }^{2}$ All three occur in only $39 \%$ of patients with the sensorineural hearing loss most commonly manifested. ${ }^{3}$ It leaves the patient almost totally deaf. Later, severe dementia, anosmia, bladder disturbance (26\%) and hyperreflexia (24\%) occur. Our patient presented with cerebellar dysfunction and hearing loss.

As with all acute subarachnoid haemorrhage (SAH), the CSF demonstrates increased protein (100\%) and is xanthochromic (75\%) but can be normal as chronic subarachnoid haemorrhage can occur intermittently. ${ }^{3}$

The eighth cranial nerve is commonly affected as it has a long segment exposed to $\mathrm{CSF}^{4}$ and is particularly well lined by myelin that is supported by haemosiderin-sensitive microglial cells. In addition, the eighth cranial nerve lies in the pontine cistern which is a large pool of CSF subjected to greater CSF flow. The result is greater axonal damage. The same theory applies to the susceptibility of the olfactory nerves to increased iron load. The second cranial nerve is entirely within the CNS and is thus spared from the damaging effects of this process.

This occurs three times more commonly in males and has a broad age range (14-77 years) of presentation.

The cause of the recurrent SAH is determined on imaging in only $50 \%$ of cases $^{3}$ and includes dural pathology, postsurgical CSF cavity with neovascularity, bleeding central nervous system (CNS) tumours (ependymoma, oligodendroglioma and astrocytoma), vascular malformations (arteriovenous malformations and cavernous malformation near the brain's surface), aneurysms, and in the spinal cord - tumours, arteriovenous malformations and traumatic nerve root avulsion. ${ }^{2}$

Pathologically there is brown discoloration of the leptomeninges of the cerebellum (especially the vermis), basal frontal lobe, olfactory bulb, temporal cortex, brainstem, cranial nerves, spinal cord, nerve roots, ependyma and subpial tissue of $<3 \mathrm{~mm}$ deep. ${ }^{1}$ The vermis of the cerebellum is particularly susceptible due to its close anatomical relation to the roof of the fourth ventricle and the compartmentalisation of CSF, therefore resulting in consistent increased exposure to CSF contents.

MRI can be used to make the diagnosis of superficial siderosis. The MRI GRE is the most sensitive, ${ }^{1}$ and demonstrates thick dark signal lining the nervous system surfaces as a susceptibility artifact due to haemosiderin. This shows no enhancement. Because of the paramagnetic nature of haemosiderin, T1- and T2-weighted sequences demonstrate a blackened surface. ${ }^{5}$ The seventh and eighth cranial nerves appear darker and thicker than normal.

Fluid attenuated inversion recovery (FLAIR) imaging suggests the diagnosis as the darker surface of the CNS and should prompt one to perform the GRE sequence. $^{3}$

Once the diagnosis is made a search for the cause is indicated and includes contrast administration, MRA if an aneurysm is suspected and whole spine MRI.

The role of MRI is in the diagnosis of superficial siderosis, in terms of the easy detection of blood degradation products by GRE compared with computed tomography (CT), detecting a potentially treatable condition and avoiding unnecessary searches for other causes of hearing loss and ataxia. CT scan is relatively insensitive to the haemosiderin and may be suggested by a slightly hyperdense rim over the brain surface 


\section{CASE REPORT}

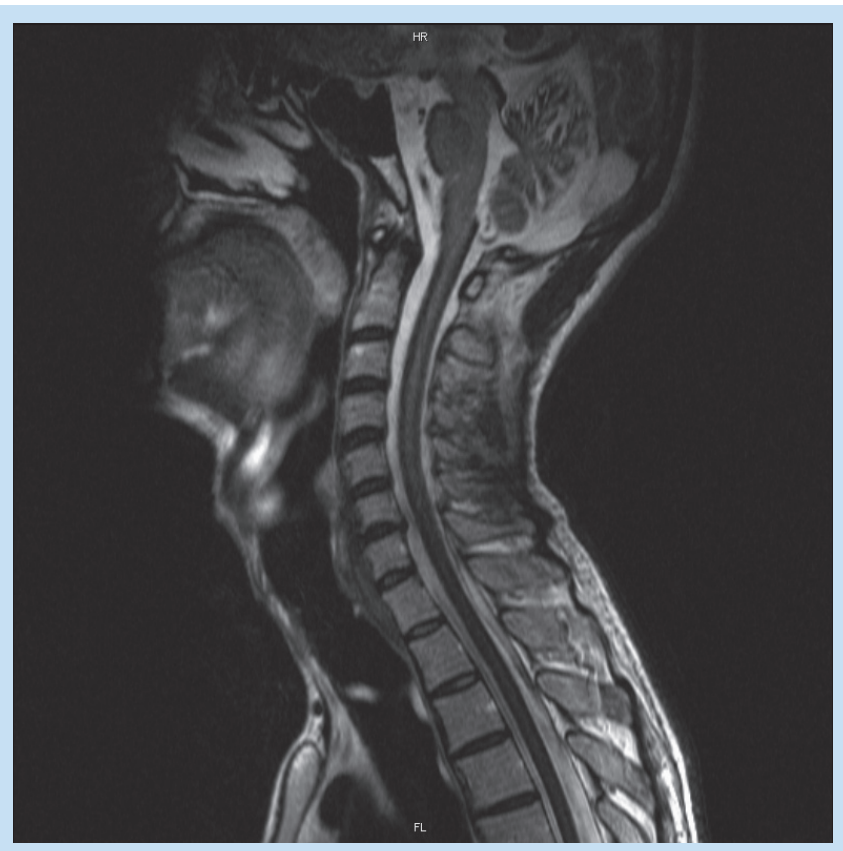

Fig. 5. MRI - sagittal T2W image demonstrating hypointensity on the pial surface of the spinal cord indicative of haemosiderin deposition.

if the iron load is heavy. ${ }^{3}$ It may demonstrate vermian atrophy and the underlying bleeding source.

The differential diagnosis includes: MRI sequence artifact seen on some but not all sequences, normal or abnormal surface veins, neurocutaneous melanosis or meningioangiomatosis. ${ }^{1}$

Neurocutaneous melanosis is a congenital syndrome consisting of benign pigmented cell tumour of the leptomeninges. It is suggested by the presence of a large congenital melanocytic nevus. Meningoangiomatosis is a rare benign focal lesion of the cerebellum and underlying cortex. The locality of the lesion, mixed signal characteristics and occasional contrast enhancement differentiates it from the diffuse nature of superficial siderosis.

Treatment is to treat the source of bleeding. There has been little success with iron or copper chelators as sole therapy.

\section{Conclusion}

The imaging findings of superficial siderosis are a reflection of the recurrent subarachnoid haemorrhage. Careful inspection of the brain surface on T2 and FLAIR should prompt one to further imaging with GRE, contrast administration and MRA.

1. Osborn AG, Blaser SI, Salzman KL, et al. Diagnostic Imaging: Brain. Manitoba: Amirsys, 2004.

2. Feamley JM, Stevens JM, Rudge P. Superficial siderosis of the central nervous system. Brain 1995; 118: 1051-1066.

3. Levy M, Turtzo C, Llinas RH. Superficial siderosis: a case report and review of the literature. Nature Clinical Practice Neurology 2007; 3: 54-58.

4. Ushio M, Lwasaki S, Sugasawa K, et al. Superficial siderosis causing retrolabyrinthine involvement in both cochlear and vestibular branches of the 8th cranial nerve. Acta Oto-Laryngologica 126; 9: 997-1000.

5. Messori A, di Bella P, Herber N, et al. The importance of suspecting superficial siderosis of the central nervous system in clinical practice. J Neurol Neurosurg Psychiatry 2004; 75: 188-190. 